\title{
Partitioning the vertex set of $G$ to make $G \square H$ an efficient open domination graph
}

\author{
Tadeja Kraner Šumenjak ${ }^{1,2}$ Iztok Peterin ${ }^{2,3 *}$ Douglas F. Rall ${ }^{4 \dagger}$ Aleksandra \\ Tepeh $^{3,5 \ddagger}$ \\ 1 University of Maribor, FKBV, Hoče, Slovenia \\ 2 IMFM, Ljubljana, Slovenia \\ 3 University of Maribor, FEECS, Maribor, Slovenia \\ 4 Furman University, Greenville, SC, USA \\ 5 Faculty of Information Studies, Novo Mesto, Slovenia
}

received $18^{\text {th }}$ Aug. 2015, revised $26^{\text {th }}$ May 2016, accepted $31^{\text {st }}$ May 2016.

\begin{abstract}
A graph is an efficient open domination graph if there exists a subset of vertices whose open neighborhoods partition its vertex set. We characterize those graphs $G$ for which the Cartesian product $G \square H$ is an efficient open domination graph when $H$ is a complete graph of order at least 3 or a complete bipartite graph. The characterization is based on the existence of a certain type of weak partition of $V(G)$. For the class of trees when $H$ is complete of order at least 3 , the characterization is constructive. In addition, a special type of efficient open domination graph is characterized among Cartesian products $G \square H$ when $H$ is a 5 -cycle or a 4-cycle.
\end{abstract}

Keywords: efficient open domination, Cartesian product, vertex labeling, total domination

\section{Introduction}

The domination number of a graph $G$ is a classical invariant in graph theory. It is the minimum cardinality of a set $S$ of vertices for which the union of the closed neighborhoods centered in vertices of $S$ is the entire vertex set of $G$. Hence, each vertex of $G$ is either in $S$ or is adjacent to a vertex in $S$. In other words, we can say that vertices of $S$ control each vertex outside of $S$. A classical question in such a situation is: who controls the vertices of $S$ ? One possible solution to this dilemma is total domination. A set $D \subseteq V(G)$ is a total dominating set of $G$ if every vertex of $G$ is adjacent to a vertex of $D$. (Hence, vertices of $D$ are also controlled by $D$.)

\footnotetext{
*Partially supported by the Ministry of Science of Slovenia under the grant P1-0297.

$\dagger$ This author was supported by the grant "Internationalisation-a pillar of development of University of Maribor." and by a grant from the Simons Foundation (\#209654 to Douglas F. Rall).

${ }^{\ddagger}$ This author was partially supported by Slovenian research agency ARRS, program no. P1-00383, project no. L1-4292, and Creative Core-FISNM-3330-13-500033.
}

Distributed under a Creative Commons Attribution 4.0 International License 
A natural question for a graph $G$ is whether we can find a total dominating set $D$ such that the union of the open neighborhoods of the vertices in $D$ is $V(G)$ but so that these open neighborhoods also form a partition of $V(G)$. The concept has been presented under the names total perfect codes Cockayne et al. (1993), efficient open domination Gavlas and Schultz (2002) and exact transversals Cowen et al. (2007). In the present work we follow the terminology of efficient open domination, and we say that a graph $G$ is an efficient open domination graph if $G$ has a total dominating set $D$ such that the open neighborhoods of the vertices of $D$ form a partition of $V(G)$. A similar concept for ordinary domination was first investigated by Biggs (1973) and Kratochvíl (1986). They call a graph 1-perfect if it contains a perfect code, that is, a set of vertices whose closed neighborhoods partition the vertex set.

The problem of establishing whether a graph $G$ is an efficient open domination graph is an NPcomplete problem; see Gavlas et al. (2003); McRae (1984). Gavlas et al. (2003) gave a recursive characterization of the class of efficient open domination trees. Gavlas and Schultz (2002) presented various properties of efficient open domination graphs. The efficient open domination graphs that are also Cayley graphs were studied by Tamizh Chelvam and Mutharasu (2012) and efficient open domination grid graphs by Cowen et al. (2007); Dejter (2008); Klostermeyer and Goldwasser (2006). Moreover, Abay-Asmerom et al. (2008) characterized those direct product graphs that are efficient open domination graphs.

Several graph products have been investigated in the last few decades and a rich theory involving the structure and recognition of classes of these graphs has emerged Hammack et al. (2012). The most studied graph products are the Cartesian, strong, direct, and lexicographic. These four are also called the standard products. One approach to graph products is to deduce properties of a product with respect to (the same) properties of its factors. See a short collection of these types involving total domination and perfect codes in Dorbec et al. (2006); Gravier (2002); Henning and Rall (2005); Ho (2008); Jerebic et al. (2005); Klavžar et al. (2006); Kuziak et al. (2014a,b); Mekiš (2010); Rall (2005). The domination related questions on the Cartesian product seems to be the most problematic among the standard products. We just mention Vizing's conjecture, which says that the domination number of a Cartesian product is at least the product of the domination numbers of the two factors. Settling this conjecture is one of the most challenging problems in the area of domination (see the recent survey on Vizing's conjecture Brešar et al. (2012)). Efficient open domination is no exception, which could be the reason it has not been studied intensively yet in the Cartesian product setting. Other than the results on grid graphs mentioned above, a step forward in this direction was made only recently by Kuziak et al. (2014b) where some special types of Cartesian products were considered. In the same paper complete descriptions of efficient open domination graphs among lexicographic and strong products of graph were given.

The aim of this paper is to show how the problem of finding efficient open domination graphs among Cartesian products can be approached by partitioning the vertex set of one factor. In the next section we set the context by supplying needed definitions and previous results in this area. In Section 3 we prove that for $r \geq 3$, the graph $G \square K_{r}$ has an efficient open dominating set if and only if $V(G)$ has a weak partition that satisfies certain properties. This provides a way to construct graphs with efficient open dominating sets in this family of Cartesian products. In addition we give a structural characterization of the trees $T$ such that $T \square K_{r}$ has an efficient open dominating set. Section 4 addresses this weak partition approach to graphs of diameter 2. 


\section{Definitions and previous results}

Throughout the article we consider only finite, simple graphs. For most common graph theory notation and definitions we follow the book by Hammack et al. (2012). In particular, our definitions and notation for open $(N(v))$ and closed $(N[v])$ neighborhoods of a vertex $v$, for distance $\left(d_{G}(u, v)\right)$ between a pair of vertices and for the diameter $(\operatorname{diam}(G))$ of a graph are the same as theirs. The distance $d_{G}(e, v)$ between an edge $e$ and a vertex $v$ in $G$ is the shortest distance between $v$ and the two end vertices of $e$, while the distance $d_{G}\left(e_{1}, e_{2}\right)$ between edges $e_{1}$ and $e_{2}$ is the shortest distance between the end vertices of $e_{1}$ and the end vertices of $e_{2}$. In general, for nonempty subsets $P$ and $Q$ of $V(G)$, the distance $d_{G}(P, Q)$ between them is the shortest distance between a vertex from $P$ and a vertex from $Q$. A weak partition of a set $X$ is a collection of pairwise disjoint subsets of $X$ whose union is $X$. We emphasize that, in contrast to a partition, members of a weak partition are allowed to be empty. The subgraph induced by a subset $S$ of $V(G)$ is denoted by $\langle S\rangle$. A matching in $G$ is any (possibly empty) set of independent edges. If $r$ is a positive integer, then the vertex set of each of the graphs $P_{r}, K_{r}$, and $C_{r}$ (if $r>2$ ) will be the interval $[r]$ defined by $[r]=\{1, \ldots, r\}$.

Since this present work concerns total domination on Cartesian products, we include several of the important definitions here for the sake of completeness. We say that a vertex $x$ of $G$ dominates a vertex $y$ (equivalently, $y$ is dominated by $x$ ) if $y \in N(x)$. A subset $D$ of $V(G)$ is a total dominating set of $G$ if each vertex in $G$ is dominated by at least one vertex in $D$. The total domination number of a graph $G$ is the minimum cardinality of a total dominating set of $G$ and is denoted by $\gamma_{t}(G)$. The Cartesian product, $G \square H$, of graphs $G$ and $H$ is a graph with $V(G \square H)=V(G) \times V(H)$. Two vertices $(g, h)$ and $\left(g^{\prime}, h^{\prime}\right)$ are adjacent in $G \square H$ whenever $\left(g g^{\prime} \in E(G)\right.$ and $\left.h=h^{\prime}\right)$ or $\left(g=g^{\prime}\right.$ and $\left.h h^{\prime} \in E(H)\right)$. For a fixed $h \in V(H)$ we call $G^{h}=\{(g, h) \in V(G \square H): g \in V(G)\}$ a $G$-layer in $G \square H$. Similarly, an $H$-layer ${ }^{g} H$ for a fixed $g \in V(G)$ is defined as ${ }^{g} H=\{(g, h) \in V(G \square H): h \in V(H)\}$. Notice that the subgraph of $G \square H$ induced by a $G$-layer or an $H$-layer is isomorphic to $G$ or $H$, respectively. The map $p_{G}: V(G \square H) \rightarrow V(G)$ defined by $p_{G}((g, h))=g$ is called a projection map onto $G$. Similarly, we define $p_{H}$ as the projection map onto $H$. Projections are defined as maps between vertices, but frequently it is more useful to see them as maps between graphs.

A graph $G$ is an efficient open domination graph (shortly an EOD-graph) if there exists a set $D$, called an efficient open dominating set (shortly an EOD-set), for which $\bigcup_{v \in D} N(v)=V(G)$ and $N(u) \cap$ $N(v)=\emptyset$ for every pair $u$ and $v$ of distinct vertices of $D$. Note that two different vertices of an EOD-set are either adjacent or at distance at least three. It is easy to see that the path $P_{n}$ is an EOD-graph if and only if $n \not \equiv 1(\bmod 4)$, while the cycle $C_{n}$ is an EOD-graph if and only if $n \equiv 0(\bmod 4)$. Let $G$ and $H$ be graphs such that $G \square H$ is an EOD-graph with an EOD-set $D$. Note that the projection of an edge in $\langle D\rangle$ onto $G$ is either a vertex or an edge. When the projection of every edge in $\langle D\rangle$ onto $G$ is an edge, we say that $D$ is a parallel EOD-set with respect to $G$. A Cartesian product that contains a parallel EOD-set with respect to one of its factors is called a parallel EOD-graph.

Among the class of nontrivial Cartesian products several infinite families of EOD-graphs have been found. In Cowen et al. (2007); Klostermeyer and Goldwasser (2006) the authors investigated EOD-graphs among the grid graphs (that is, Cartesian products of paths). Results from both papers are merged in the following characterization.

Theorem 2.1 Cowen et al. (2007); Klostermeyer and Goldwasser (2006) Let $t \geq r \geq 3$. The grid graph $P_{r} \square P_{t}$ is an EOD-graph if and only if $r$ is an even number and $t \equiv x(\bmod r+1)$ for some $x \in\{1, r-2, r\}$. 
Some partial results on EOD-graphs in the family of torus graphs (Cartesian products of cycles) were presented by Dejter (2008), by characterizing only those with a parallel EOD-set (there referred to as a parallel total perfect code).

Theorem 2.2 Dejter (2008) The Cartesian product $C_{r} \square C_{t}$ has a parallel EOD-set if and only if $r$ and $t$ are multiples of four.

Kuziak et al. (2014b) recently continued with the study of EOD-graphs among tori and cylinders (Cartesian product of a path and a cycle).

Proposition 2.3 Kuziak et al. (2014b) Let $t \geq 4$. The torus $C_{4} \square C_{t}$ is an EOD-graph if and only if $t \equiv 0$ $(\bmod 4)$.

In addition, they proved that $C_{r} \square C_{t}$ is not an EOD-graph if $r \in\{3,5,6,7\}$ and $t \geq r$. Based on the above observations they posed the following conjecture.

Conjecture 2.4 Kuziak et al. (2014b) Let $r$ and $t$ be integers such that $r \geq 3$ and $t \geq 3$. The torus $C_{r} \square C_{t}$ is an EOD-graph if and only if $r \equiv 0(\bmod 4)$ and $t \equiv 0(\bmod 4)$.

The same authors characterized the graphs $G$ for which $G \square K_{2}$ is an EOD-graph. In order to do this they introduced the so-called zig-zag graphs, Kuziak et al. (2014b). Let $G$ be a graph on at least three vertices and $E^{\prime}=\left\{e_{1}, \ldots, e_{k}\right\}$ a subset of $E(G)$, where $e_{i}=u_{i} v_{i}$ for every $i \in[k]$, with the following properties:

(i) $N\left(u_{i}\right) \cap N\left(v_{i}\right)=\emptyset$;

(ii) $d_{G}\left(e_{i}, e_{j}\right) \geq 2$ for $1 \leq i<j \leq k$;

(iii) for every $x \in V(G)-\left\{u_{i}, v_{i}: i \in[k]\right\}$ there exist unique $j$ and $\ell, j \neq \ell$, such that $d_{G}\left(x, e_{j}\right)=$ $d_{G}\left(x, e_{\ell}\right)=1$

(iv) for every sequence $e_{i_{1}}, \ldots, e_{i_{j}}$ of distinct edges with $j>2$ and with $d_{G}\left(e_{i_{\ell}}, e_{i_{\ell+1}(\bmod j)}\right)=2$ for $\ell \in\{1, \ldots, j\}, j$ must be an even number.

We call $E^{\prime}$ a zig-zag set of $G$ and, if there exists a zig-zag set in $G$, we call $G$ a zig-zag graph.

Theorem 2.5 Kuziak et al. (2014b) If $G$ is a zig-zag graph, then $G \square K_{2}$ is an EOD-graph.

Not all EOD-graphs among $G \square K_{2}$ are given by the above theorem. Kuziak et al. observed that for a description of all EOD-graphs among Cartesian products of graphs with $K_{2}$, a certain combination of zig-zag graphs and 1-perfect graphs is needed (see Kuziak et al. (2014b) for details).

One can observe that for $r>2$, every EOD-set in $G \square K_{r}$ is a parallel EOD-set with respect to $G$. Namely, if an edge induced by two vertices of a vertex subset $A$ of $G \square K_{r}$ projects to a single vertex $g \in V(G)$, then the layer ${ }^{g} K_{r}$ contains a vertex that is dominated more than once by $A$. This observation led to the idea of how to approach the problem of finding EOD-graphs among $G \square K_{r}$ for $r>2$. This is presented in the next section. 


\section{$3 G \square K_{r}$ for $r>2$}

In order to obtain a characterization of EOD-graphs among $G \square K_{r}, r>2$, we introduce a new concept, based on a weak partition of the vertex set of $G$. As we will see in later sections, a modification of this concept can be used for the construction of EOD-graphs that are Cartesian products $G \square H$ where $H$ belongs to several other special classes of graphs.

Let $r$ be an integer larger than 1 . We call a graph $G$ a $K_{r}$-amenable graph if there exists a weak partition $\left\{V_{0}, V_{1}, \ldots, V_{r}\right\}$ of $V(G)$, such that

(A) if $x \in V_{0}$, then $\left|N(x) \cap V_{i}\right|=1$ for every $i \in[r]$,

(B) $\left\langle V_{i}\right\rangle$ is a matching in $G$ for every $i \in[r]$,

(C) $\left\langle V_{1} \cup \cdots \cup V_{r}\right\rangle$ is a matching in $G$.

For the sake of clarity in the above definition we emphasize that the induced subgraphs $\left\langle V_{i}\right\rangle$ and $\left\langle V_{1} \cup \cdots \cup V_{r}\right\rangle$ do not contain any edges other than those in their perfect matchings.

We first prove that $K_{2}$-amenable graphs do not differ from zig-zag graphs.

Theorem 3.1 A graph $G$ is a $K_{2}$-amenable graph if and only if $G$ is a zig-zag graph.

Proof: Let $G$ be a $K_{2}$-amenable graph with a weak partition $\left\{V_{0}, V_{1}, V_{2}\right\}$ of $V(G)$ that satisfies conditions (A), (B) and (C). We will show that $E^{\prime}=\left\langle V_{1} \cup V_{2}\right\rangle$ is a zig-zag set of $G$ by demonstrating that conditions (i) $-(i v)$ hold. Since $\left\langle V_{1} \cup V_{2}\right\rangle,\left\langle V_{1}\right\rangle$ and $\left\langle V_{2}\right\rangle$ are matchings, $E^{\prime}$ is a set of edges $\left\{e_{1}, \ldots, e_{k}\right\}$. By the same argument we derive that $d_{G}\left(e_{i}, e_{j}\right) \geq 2$ for $i \neq j$, and thus (ii) holds. Let $e_{i}=u_{i} v_{i}$ for every $i \in[k]$. If $x \in N\left(u_{i}\right) \cap N\left(v_{i}\right)$ for some $i \in[k]$, then $x \in V_{0}$ by the matching argument again. But this contradicts condition (A) since $\left|N(x) \cap V_{i}\right| \geq 2$ in this case. Hence $(i)$ also holds. If $x \in$ $V(G)-\left\{u_{i}, v_{i}: i \in[k]\right\}$, then $x \in V_{0}$. By (A) we have that $\left|N(x) \cap V_{i}\right|=1$ for every $i \in\{1,2\}$, which implies the existence of exactly two different edges $e_{j}$ and $e_{\ell}$ of $E^{\prime}$ with $d_{G}\left(x, e_{j}\right)=d_{G}\left(x, e_{\ell}\right)=$ 1. This proves $(i i i)$. To prove $(i v)$, let $e_{i_{1}}, e_{i_{2}}, \ldots, e_{i_{j}}, j>2$, be a sequence of distinct edges with $d_{G}\left(e_{i_{\ell}}, e_{i_{\ell+1}(\bmod j)}\right)=2$ for $\ell \in[j]$. In addition, let $x_{\ell}$ be a common neighbor of $e_{i_{\ell}}$ and $e_{i_{\ell+1}(\bmod j)}$. As before, $x_{\ell} \in V_{0}$ for every $\ell \in[j]$. Without loss of generality, suppose the end-vertices of the edge $e_{i_{1}}$ belong to $V_{1}$. By condition (A) for the vertex $x_{1}$, the end-vertices of $e_{i_{2}}$ belong to $V_{2}$. The same argument for the vertex $x_{2}$ implies that the end-vertices of $e_{i_{3}}$ belong to $V_{1}$. Continuing this way, we get a zig-zag pattern for the end-vertices of $e_{i_{1}}, e_{i_{2}}, \ldots, e_{i_{j}}$. If $j$ is an odd number, then the end-vertices of $e_{i_{1}}$ and $e_{i_{j}}$ are both in $V_{1}$, which gives a contradiction with condition (A) for the vertex $x_{j}$. Thus $j$ is an even number and $(i v)$ holds as well.

Now let $G$ be a zig-zag graph with a zig-zag set $E^{\prime}=\left\{e_{1}, \ldots, e_{k}\right\}$ where $e_{i}=u_{i} v_{i}$. We set $V_{0}=$ $V(G)-\left\{u_{i}, v_{i}: i \in[k]\right\}$. Observe that $E^{\prime}$ can be partitioned as $E^{\prime}=E_{1} \cup \cdots \cup E_{t}$ such that for each $i \in[t]$, the following holds. The set $E_{i}$ is a maximal set of edges such that between any two distinct edges $e_{j}$ and $e_{n}$ from $E_{i}$ there exists a sequence $e_{j}=e_{j_{0}}, e_{j_{1}}, \ldots, e_{j_{\ell}}=e_{n}, \ell \geq 1$, of distinct edges where the distance between two consecutive edges in this sequence is 2 . Such a sequence is called a 2 -step sequence of length $\ell$.

Observe that there exists a partition of $E^{\prime}=E_{1} \cup \cdots \cup E_{t}$, such that $E_{i}$, for every $i \in[t]$, consists of a maximal set of edges such that between any two distinct edges $e_{j}$ and $e_{k}$ from $E_{i}$ there exists a sequence $e_{j}=e_{j_{0}}, e_{j_{1}}, \ldots, e_{j_{\ell}}=e_{k}, \ell \geq 1$, of distinct edges such that the distance between two consecutive edges 
in this sequence is 2 (we call such sequence a 2-step sequence of length $\ell$ ). Now, in $E_{i}$ fix an arbitrary edge $e$. For an arbitrary edge $f$ in $E_{i}$ there exists a 2-step sequence between $e$ and $f$. Property $(i v)$ implies that the lengths of all different 2-step sequences between $e$ and $f$ are of the same parity. Thus, edges of $E_{i}$ can be partitioned into two sets $E_{i}^{1}$ and $E_{i}^{2}$. The set $E_{i}^{1}$ consists of $e$ and all edges $f$ for which the length of a 2-step sequence between $e$ and $f$ is even, and $E_{i}^{2}=E_{i}-E_{i}^{1}$. For every $i \in[t]$ let $V_{i}^{1}$ denote the set of end-vertices of edges in $E_{i}^{1}$, and $V_{i}^{2}$ the set of end-vertices of edges in $E_{i}^{2}$. Finally, let $V_{1}=V_{1}^{1} \cup \cdots \cup V_{t}^{1}$ and $V_{2}=V_{1}^{2} \cup \cdots \cup V_{t}^{2}$.

We will show that $\left\{V_{0}, V_{1}, V_{2}\right\}$ is a weak partition of $V(G)$ satisfying conditions (A), (B) and (C). Properties (B) and (C) clearly follow, since $d_{G}\left(e_{i}, e_{j}\right) \geq 2$ for every pair $e_{i}, e_{j} \in E^{\prime}$. To prove (A) let $x \in V_{0}$. By $($ iii $)$ there exist exactly two different edges $e_{p}, e_{r} \in E^{\prime}$ such that $d_{G}\left(x, e_{p}\right)=1=d_{G}\left(x, e_{r}\right)$. Note that $e_{p}$ and $e_{r}$ belong to the same $E_{i}$ in the partition of $E^{\prime}$. Recall that we have fixed the edge $e \in E_{i}$. If a 2-step sequence between $e$ and $e_{p}$ and a 2-step sequence between $e$ and $e_{r}$ have the same parity, then we obtain a contradiction with $(i v)$. Hence, end-vertices of one edge, say $e_{p}$, belong to $V_{1}$, and end-vertices of $e_{r}$ belong to $V_{2}$. Since, in addition, $N\left(u_{i}\right) \cap N\left(v_{i}\right)=\emptyset$, by $(i)$ for every $i$ we have $\left|N(x) \cap V_{1}\right|=1=\left|N(x) \cap V_{2}\right|$ and condition (A) holds.

Theorem 3.2 Let $r$ be a positive integer such that $r>2$ and let $G$ be a graph. The Cartesian product $G \square K_{r}$ is an EOD-graph if and only if $G$ is a $K_{r}$-amenable graph.

Proof: Let $G$ be a $K_{r}$-amenable graph with corresponding weak partition $\left\{V_{0}, \ldots, V_{r}\right\}$ of $V(G)$. We define a subset $D$ of $V\left(G \square K_{r}\right)$ by $D=\left\{(g, i): i \in[r]\right.$ and $\left.g \in V_{i}\right\}$. It follows that $D$ contains at most one vertex from each $K_{r}$-layer. To prove that $G \square K_{r}$ is an EOD-graph we will show that every vertex of $G \square K_{r}$ is dominated by exactly one vertex of $D$. Let $i \in[r]$ and let $g \in V(G)$. First, assume that $g \in V_{0}$. By (A), the vertex $g$ has a unique neighbor $x_{i}$ in $V_{i}$. Consequently, $(g, i)$ is adjacent to $\left(x_{i}, i\right)$ and $\left(x_{i}, i\right) \in D$. Moreover, by the uniqueness of $x_{i}$, no other vertex of $D$ dominates $(g, i)$. Now assume that $g \in V_{i}$. Since $\left\langle V_{i}\right\rangle$ is a perfect matching, $g$ has a unique neighbor $g^{\prime}$ in $V_{i}$. It follows that $\left(g^{\prime}, i\right) \in D$ and that $\left(g^{\prime}, i\right)$ is the only neighbor of $(g, i)$ in $D$. Finally, assume that $g \in V_{j}$ for some $j \in[r]$ such that $j \neq i$. By the definition of $D$ this implies that $\{(g, j)\}=D \cap{ }^{g} K_{r}$. In addition, since (B) and (C) hold, $(g, i)$ has no neighbor in $G^{i} \cap D$. The result is that $(g, i)$ is dominated by exactly one vertex, namely $(g, j)$, of $D$. Consequently, $D$ is an EOD-set of $G \square K_{r}$ and $G \square K_{r}$ is an EOD-graph.

To prove the converse, suppose that $G \square K_{r}$ is an EOD-graph with an EOD-set $D$. For $i \in[r]$ let $V_{i}=\{v \in V(G):(v, i) \in D\}$, and let $V_{0}=V(G)-\left(V_{1} \cup \cdots \cup V_{r}\right)$. As we observed in Section 2, $D$ is necessarily parallel with respect to $G$. This means that every ${ }^{v} K_{r}$ contains at most one vertex of $D$, and we thus infer that $\left\{V_{0}, V_{1}, \ldots, V_{r}\right\}$ is a weak partition of $V(G)$. We prove that conditions (A), (B), and (C) of the definition of $K_{r}$-amenable hold. If condition (A) is not satisfied, then there exist $x \in V_{0}$ and $i \in[r]$, such that $\left|N(x) \cap V_{i}\right|=0$ or $\left|N(x) \cap V_{i}\right|>1$. In the first case $(x, i)$ is not dominated by any vertex of $D$, and in the second case $(x, i)$ is dominated by more than one vertex of $D$. Both cases are in contradiction with the assumption that $D$ is an EOD-set of $G \square K_{r}$. Hence, the weak partition $\left\{V_{0}, V_{1}, \ldots, V_{r}\right\}$ satisfies property (A). Let $i \in[r]$ and let $g \in V_{i}$. Since $\left|D \cap{ }^{g} K_{r}\right| \leq 1$ and $(g, i)$ has exactly one neighbor in $D$, it follows that $\left|N(g) \cap\left(V_{1} \cup \cdots \cup V_{r}\right)\right|=1=\left|N(g) \cap V_{i}\right|$. Hence, both (B) and (C) hold. Therefore, $G$ is a $K_{r}$-amenable graph.

Let $r$ be an integer larger than 1. In the rest of this section we present a recursive description of the family of all $K_{r}$-amenable trees. The following construction generalizes the construction of zig-zag trees (that is, $K_{2}$-amenable trees) from Kuziak et al. (2014b). We will denote by $K_{1, r}^{+}$the tree of order $2 r+1$ 
obtained from the star $K_{1, r}$ by subdividing each edge exactly once. It is clear that $K_{1, r}^{+}$is a $K_{r}$-amenable tree, and the corresponding partition of $V\left(K_{1, r}^{+}\right)$is unique up to a permutation of $[r]$. We now define an infinite family $\mathcal{T}_{r}$ of trees. Each member of $\mathcal{T}_{r}$ will have a weak partition $\left\{V_{0}, V_{1}, \ldots, V_{r}\right\}$ of its vertex set associated with it.

Suppose that $T^{\prime}$ is a tree of order $n$ such that $\left\{V_{0}^{\prime}, V_{1}^{\prime}, \ldots, V_{r}^{\prime}\right\}$ is a weak partition of $V\left(T^{\prime}\right)$ and that $T^{\prime \prime}$ is a tree of order $m$ such that $\left\{V_{0}^{\prime \prime}, V_{1}^{\prime \prime}, \ldots, V_{r}^{\prime \prime}\right\}$ is a weak partition of $V\left(T^{\prime \prime}\right)$.

We say that a tree $T$ of order $n+m-2$ is obtained from $T^{\prime}$ and $T^{\prime \prime}$ by a Type-a construction if $T$ is isomorphic to the tree formed by choosing any $i \in[r]$, any edge $u_{i}^{\prime} v_{i}^{\prime}$ in $\left\langle V_{i}^{\prime}\right\rangle$, any edge $u_{i}^{\prime \prime} v_{i}^{\prime \prime}$ in $\left\langle V_{i}^{\prime \prime}\right\rangle$ and then identifying the vertices $u_{i}^{\prime}$ with $u_{i}^{\prime \prime}$ (now called $u_{i}$ ) and $v_{i}^{\prime}$ with $v_{i}^{\prime \prime}$ (now called $v_{i}$ ) to obtain the edge $u_{i} v_{i}$ in $T$. The associated weak partition $\left\{V_{0}, V_{1}, \ldots, V_{r}\right\}$ of $V(T)$ is defined by $V_{j}=V_{j}^{\prime} \cup V_{j}^{\prime \prime}$ if $j \neq i$, and $V_{i}=\left(V_{i}^{\prime} \cup V_{i}^{\prime \prime} \cup\left\{u_{i}, v_{i}\right\}\right)-\left\{u_{i}^{\prime}, v_{i}^{\prime}, u_{i}^{\prime \prime}, v_{i}^{\prime \prime}\right\}$.

A tree $S$ of order $n+m$ is obtained from $T^{\prime}$ and $T^{\prime \prime}$ by a Type-b construction if $S$ is isomorphic to the tree formed from the union of $T^{\prime}$ and $T^{\prime \prime}$ by adding an edge $x y$ for some $x \in V_{0}^{\prime}$ and some $y \in V_{0}^{\prime \prime}$. The associated weak partition $\left\{V_{0}, V_{1}, \ldots, V_{r}\right\}$ of $V(S)$ is given by $V_{i}=V_{i}^{\prime} \cup V_{i}^{\prime \prime}$ for $0 \leq i \leq r$.

The family $\mathcal{T}_{r}$ is defined recursively as follows. A tree $T$ belongs to $\mathcal{T}_{r}$ if and only if $T=K_{1, r}^{+}$with its partition as indicated above or $T$ can be obtained from smaller trees in $\mathcal{T}_{r}$ by a finite sequence of Type-a or Type-b constructions.

Theorem 3.3 Let $r$ be an integer such that $r \geq 2$. The path of order 2 is $K_{r}$-amenable. If $T$ is a tree of order more than 2 , then $T$ is a $K_{r}$-amenable graph if and only if $T \in \mathcal{T}_{r}$.

Proof: Let $r$ be an integer such that $r \geq 2$. For the path of order 2 , let $V_{1}=V\left(P_{2}\right), V_{0}=\emptyset=V_{i}$ for $2 \leq i \leq r$. This weak partition $\left\{V_{0}, V_{1}, \ldots, V_{r}\right\}$ satisfies the definition showing that $P_{2}$ is a $K_{r}$-amenable graph. For the remainder of this proof we assume that all trees under consideration have order at least 3. As noted above, the tree $K_{1, r}^{+}$is a $K_{r}$-amenable tree. One can conclude directly from the definitions that if $T^{\prime}$ and $T^{\prime \prime}$ are both $K_{r}$-amenable trees, then a tree obtained from $T^{\prime}$ and $T^{\prime \prime}$ by a Type-a or a Type-b construction is also a $K_{r}$-amenable graph. Thus, it follows by induction (on the number of Type-a and Type-b constructions) that every member of $\mathcal{T}_{r}$ is a $K_{r}$-amenable graph.

Conversely, let $T$ be a $K_{r}$-amenable tree of order at least 3 with a corresponding weak partition $\left\{V_{0}, V_{1}, \ldots, V_{r}\right\}$ and let $k=\left|V_{0}\right|$. Since $T$ has order at least 3, it follows from the definition that $k \geq 1$. We use induction on $k$ to show that $T \in \mathcal{T}_{r}$. Let $k=1$ and $V_{0}=\{v\}$. By property $(\mathrm{A}) \operatorname{deg}(v)=r$; let $N(v)=\left\{u_{1}, \ldots, u_{r}\right\}$ where $u_{i} \in V_{i}$. By (B) every $u_{i}$ has a unique neighbor $w_{i}$ in $V_{i}$ and by (C) $u_{i}$ and $w_{i}$ have no neighbors in $V_{j}$ for $j \neq i$. Moreover, $u_{i}$ and $w_{i}$ have no additional neighbors in $V_{0}$ since $k=1$. Thus, $T$ is isomorphic to $K_{1, r}^{+}$and hence $T \in \mathcal{T}_{r}$

Now suppose that $k>1$. Note that every vertex in $V_{0}$ has degree at least $r$. If there exists $v \in V_{0}$ with $\operatorname{deg}(v)>r$, then there exists $w \in V_{0} \cap N(v)$. Let $T^{\prime}$ be the component of $T-v w$ that contains $v$ and let $T^{\prime \prime}$ be the component that contains $w$. For $0 \leq i \leq r$, let $V_{i}^{\prime}=V_{i} \cap V\left(T^{\prime}\right)$ and let $V_{i}^{\prime \prime}=V_{i} \cap V\left(T^{\prime \prime}\right)$. The resulting weak partitions of $V\left(T^{\prime}\right)$ and $V\left(T^{\prime \prime}\right)$ clearly satisfy properties (A), (B) and (C), and furthermore $\left|V_{0}^{\prime}\right|<k$ and $\left|V_{0}^{\prime \prime}\right|<k$. By the induction hypothesis both $T^{\prime}$ and $T^{\prime \prime}$ belong to $\mathcal{T}_{r}$. Since $T$ is obtained from $T^{\prime}$ and $T^{\prime \prime}$ by a Type-b construction, it follows that $T \in \mathcal{T}_{r}$.

Now, suppose that all vertices of $V_{0}$ are of degree $r$ (and hence $\left\langle V_{0}\right\rangle$ contains no edges). Choose $u$ and $v$ from $V_{0}$ with the property that $d_{T}(u, v)$ is minimum among all different pairs of vertices from $V_{0}$. Clearly, $2 \leq d_{T}(u, v) \leq 3$. Let $w$ be the neighbor of $u$ on the shortest $u, v$-path in $T$. Without loss of generality we may assume that $w \in V_{1}$. By (B), $w$ has a unique neighbor, say $w^{\prime}$, in $V_{1}$. The forest $T-u w$ has two 
connected components. The component that contains $u$ is denoted by $T_{u}$ and the one that contains $v$ is denoted by $T^{\prime \prime}$. Let $T^{\prime}$ be the tree obtained from $T_{u}$ by adding vertices $t$ and $t^{\prime}$ and adding edges $u t$ and $t t^{\prime}$. Let $V_{i}^{\prime \prime}=V_{i} \cap V\left(T^{\prime \prime}\right)$ for $0 \leq i \leq r$, let $V_{1}^{\prime}=\left(V_{1} \cap V\left(T_{u}\right)\right) \cup\left\{t, t^{\prime}\right\}$, and let $V_{i}^{\prime}=V_{i} \cap V\left(T_{u}\right)$ for $i=0$ and $2 \leq i \leq r$. Properties (A), (B) and (C) clearly hold for the above defined weak partitions of $V\left(T^{\prime}\right)$ and $V\left(T^{\prime \prime}\right)$. Thus, $T^{\prime}$ and $T^{\prime \prime}$ are $K_{r}$-amenable trees. By the induction hypothesis, they are also in $\mathcal{T}_{r}$. Note that $T$ is isomorphic to the tree obtained from $T^{\prime}$ and $T^{\prime \prime}$ by a Type-a construction that identifies $t$ with $w$, and $t^{\prime}$ with $w^{\prime}$. Consequently, $T \in \mathcal{T}_{r}$.

This theorem together with Theorem 3.2 combine to give us the following characterization of those trees $T$ such that $T \square K_{r}$ is an EOD-graph for $r \geq 3$.

Corollary 3.4 Let $r$ be a positive integer larger than 2 and let $T$ be a tree. The Cartesian product $T \square K_{r}$ is an EOD-graph if and only if $T=P_{2}$ or $T \in \mathcal{T}_{r}$.

\section{$4 \quad G \square H$ with $\operatorname{diam}(H)=2$}

In this section we consider Cartesian products of graphs where (at least) one factor has diameter 2. Motivation for the study of such graphs arises from the previous section. An EOD-set of $G \square H$ that is parallel with respect to $G$ when $\operatorname{diam}(H)=2$ shares an important property with such a set in $G \square K_{r}$ for $r \geq 3$. This is given in the following lemma.

Lemma 4.1 Let $H$ be a graph of diameter 2 and let $G$ be a graph such that $G \square H$ has an EOD-set $D$. For every vertex $g$ in $G,\left|D \cap{ }^{g} H\right| \leq 2$. If in addition $D$ is parallel with respect to $G$, then $\left|D \cap{ }^{g} H\right| \leq 1$ for every $g \in V(G)$. If $\left|D \cap{ }^{g} H\right|=2$, then the two distinct vertices in $D \cap{ }^{g} H$ are adjacent.

Proof: Assume that $D$ is an EOD-set of $G \square H$ and suppose that $(g, u)$ and $(g, v)$ are distinct vertices in $D$. The graph $H$ has diameter 2, and this implies that $u v \in E(H)$ or $u$ and $v$ have a common neighbor $w$ in $H$. Since every vertex in ${ }^{g} H$ is dominated exactly once by $D$, we infer that $(g, u)$ and $(g, v)$ are adjacent, and $\left|D \cap{ }^{g} H\right| \leq 2$. It follows immediately that if $D$ is parallel with respect to $G$, then no $H$-layer can contain two members of $D$.

As we will see, finding an appropriate weak partition of vertices in $G$ will be useful in the characterization of (parallel) EOD-graphs among Cartesian products $G \square H$ where $\operatorname{diam}(H)=2$. First we show that the Cartesian product of a graph of diameter 2 and a tree on at least three vertices does not admit a parallel EOD-set with respect to the tree.

Theorem 4.2 Let $H$ be a graph with $\operatorname{diam}(H)=2$ and let $T$ be a tree. If $T$ is different than $K_{2}$, then $T \square H$ does not contain a parallel EOD-set with respect to $T$.

Proof: Let $H$ be a graph with $\operatorname{diam}(H)=2$. Suppose, in order to obtain a contradiction, that there exists a tree $T$ different than $K_{2}$, such that $T \square H$ admits a parallel EOD-set $D$ with respect to $T$.

First, we claim that $T^{h} \cap D=\emptyset$ for every non-universal vertex $h$ in $H$. If this does not hold, then there exist vertices $\left(u_{0}, h\right),\left(v_{0}, h\right) \in D$ which are adjacent in $T \square H$. Since $h$ is not universal in $H$, there is $h^{\prime} \in V(H)$ such that $d_{H}\left(h, h^{\prime}\right)=2$. Observe that $\left(u_{0}, h^{\prime}\right)$ and $\left(v_{0}, h^{\prime}\right)$ are not dominated by $\left(u_{0}, h\right)$ and $\left(v_{0}, h\right)$. Moreover, they are not dominated by any vertex in ${ }^{u_{0}} H$ and ${ }^{v_{0}} H$ (since $\operatorname{diam}(H)=2$ we have that $\left|{ }^{x} H \cap D\right| \leq 1$ for every $x \in V(T)$ by Lemma 4.1). Therefore, there exists a neighbor $u_{1}$ of $u_{0}$ and a neighbor $v_{1}$ of $v_{0}$, such that $\left(u_{0}, h^{\prime}\right)$ is dominated by $\left(u_{1}, h^{\prime}\right) \in D$ and $\left(v_{0}, h^{\prime}\right)$ is dominated by $\left(v_{1}, h^{\prime}\right) \in$ 
$D$. Moreover, since $\left(u_{1}, h^{\prime}\right),\left(v_{1}, h^{\prime}\right) \in D$, there exist $\left(u_{2}, h^{\prime}\right),\left(v_{2}, h^{\prime}\right) \in D$, where $u_{2} u_{1}, v_{2} v_{1} \in E(T)$. To dominate vertices $\left(u_{2}, h\right)$ and $\left(v_{2}, h\right)$, there must exist $\left(u_{3}, h\right),\left(v_{3}, h\right) \in D$ where $u_{3} u_{2}, v_{3} v_{2} \in E(T)$. Continuing in this way we obtain a two-way infinite walk $\ldots u_{2} u_{1} u_{0} v_{0} v_{1} v_{2} \ldots$ in $T$. Since $T$ is a tree, all vertices of this walk are pairwise different. But this is in contradiction with $T$ being finite, and the claim is proved.

We infer that $H$ has to contain universal vertices and that the projection of every edge in $\langle D\rangle$ onto $H$ is a universal vertex. Now, let $h$ be a universal vertex of $H$, such that $T^{h} \cap D \neq \emptyset$ and let $(u, h),(v, h) \in D$ be adjacent vertices. Together they dominate all vertices of ${ }^{u} H$ and ${ }^{v} H$. There also exists a non-universal vertex $h^{\prime}$ in $H$ because $\operatorname{diam}(H)=2$. Since $T$ is different than $K_{2}$, at least one of $u$ and $v$, say $u$, has a neighbor $w$ in $T$. Note that $(w, h)$ is dominated by $(u, h)$, and $\left(w, h^{\prime}\right)$ is not dominated by $(u, h)$ nor $(v, h)$. Since $T^{h^{\prime}} \cap D=\emptyset$, there exists another universal vertex $h_{1} \in V(H)$, such that $\left(w, h^{\prime}\right)$ is dominated by $\left(w, h_{1}\right) \in D$. This yields a final contradiction, since $(w, h)$ is dominated by both $(u, h)$ and $\left(w, h_{1}\right)$ from $D$, which is not possible in an EOD-set $D$.

\section{$4.1 \quad G \square K_{m, n}$}

In this subsection we give a necessary and sufficient condition on a graph $G$ such that $G \square K_{m, n}$ is an EOD-graph for $1 \leq m \leq n$. The condition will be the existence of a weak partition of $V(G)$ that satisfies very specific requirements. While it may not be easy to determine whether a given graph $G$ has such a weak partition, the requirements of the weak partition will make it straightforward to construct graphs $G$ such that $G \square K_{m, n}$ is an EOD-graph.

Since $K_{m, n}$ has diameter 2 and we are not requiring the EOD-set of $G \square K_{m, n}$ to be parallel with respect to $G$, we will refer often to Lemma 4.1. For ease of explanation we assume throughout this subsection that $1 \leq m \leq n$ and that $K_{m, n}$ has partite sets $A$ and $B$ given by $A=\{1, \ldots, m\}$ and $B=\{m+1, \ldots, m+n\}$. With this notation we let $\mathcal{C}_{m, n}$ be a weak partition of $V(G)$ containing $m n+m+n+1$ parts indexed as follows:

- $V_{0}, V_{1}, \ldots, V_{m}, V_{m+1}, \ldots, V_{m+n}$; and

- $V_{[i, m+j]}$ for $1 \leq i \leq m$ and $1 \leq j \leq n$.

We will say that $\mathcal{C}_{m, n}$ is $K_{m, n}$-amenable if it is a weak partition satisfying the following conditions.

(I) For $1 \leq i \leq m+n$, the induced subgraph $\left\langle V_{i}\right\rangle$ is a matching.

(II) For $1 \leq i \leq m$ and $m+1 \leq j \leq m+n,\left\langle V_{i} \cup V_{j}\right\rangle$ is a matching.

(III) For $1 \leq i<j \leq m$ or $m+1 \leq i<j \leq m+n$, each $x$ in $V_{i}$ has exactly one neighbor in $V_{j}$ and each $y$ in $V_{j}$ has exactly one neighbor in $V_{i}$.

(IV) If $x \in V_{[i, m+j]}$ for some $1 \leq i \leq m$ and some $1 \leq j \leq n$, then $N(x) \subseteq V_{0}$.

(V) If $x \in V_{0}$, then $\left|N(x) \cap\left(\cup_{1 \leq j \leq n} V_{[i, m+j]} \cup V_{i}\right)\right|=1$ for $1 \leq i \leq m$, and $\left|N(x) \cap\left(\cup_{1 \leq i \leq m} V_{[i, m+j]} \cup V_{m+j}\right)\right|=1$ for $1 \leq j \leq n$.

A graph $G$ will be called $K_{m, n}$-amenable if $V(G)$ has a weak partition that is $K_{m, n}$-amenable. With this definition we are now able to give a constructive characterization of those graphs $G$ such that $G \square K_{m, n}$ is an EOD-graph. 
Theorem 4.3 Let $m$ and $n$ be positive integers such that $m \leq n$ and let $G$ be a graph. The Cartesian product $G \square K_{m, n}$ is an EOD-graph if and only if $G$ is $K_{m, n}$-amenable.

Proof: Assume that $G$ is $K_{m, n}$-amenable and that $\mathcal{C}_{m, n}$ is a weak partition of $V(G)$ indexed as above and satisfying the conditions (I)-(V) in the definition above. We define a subset $D$ of $V\left(G \square K_{m, n}\right)$ by specifying its intersection with each $K_{m, n}$-layer. If $r$ is an integer such that $1 \leq r \leq m+n$ and $g \in V_{r}$, then $D \cap{ }^{g} K_{m, n}=\{(g, r)\}$. If $r$ and $s$ are integers with $1 \leq r \leq m$ and $1 \leq s \leq n$ such that $g \in V_{[r, m+s]}$, then $D \cap{ }^{g} K_{m, n}=\{(g, r),(g, m+s)\}$. Finally, if $g \in V_{0}$, then $D \cap{ }^{g} K_{m, n}=\emptyset$. Since $\mathcal{C}_{m, n}$ is a weak partition, the set $D$ is well-defined. We now show that $D$ is an EOD-set of $G \square K_{m, n}$ by showing that each vertex of $G \square K_{m, n}$ has exactly one neighbor in $D$.

Let $(x, t)$ be an arbitrary vertex in $G \square K_{m, n}$. Assume $x \in V_{0}$. Suppose first that $1 \leq t \leq m$. By (V) there exists $y \in V(G)$ such that $\{y\}=N(x) \cap\left(\cup_{1 \leq j \leq n} V_{[t, m+j]} \cup V_{t}\right)$. This implies that $(y, t) \in D$ and that $(y, t)$ dominates $(x, t)$. Furthermore, it follows from (V) and $D \cap{ }^{x} K_{m, n}=\emptyset$ that $(y, t)$ is the only neighbor of $(x, t)$ that belongs to $D$. The case $m+1 \leq t \leq m+n$ is similar. Assume next that $x \in V_{[r, m+s]}$ for some $r$ and $s$ such that $1 \leq r \leq m$ and $1 \leq s \leq n$. By the definition of $D$ we get that both $(x, r)$ and $(x, m+s)$ belong to $D$. Exactly one of these is adjacent to $(x, t)$. Combining this with property (IV) it follows that $(x, t)$ has exactly one neighbor in $D$. Finally, assume that $x \in V_{r}$ for some $r$ with $1 \leq r \leq m$. (The case $m+1 \leq r \leq m+n$ is similar.) This means that $(x, r) \in D$ and $\left|D \cap{ }^{x} K_{m, n}\right|=1$. There are three subcases to consider, namely (i) $m+1 \leq t \leq m+n$, (ii) $t=r$, and (iii) $t \neq r$ but $1 \leq t \leq m$. If $m+1 \leq t \leq m+n$, then $(x, r)$ dominates $(x, t)$ (from within the layer ${ }^{x} K_{m, n}$ ). From (I), (II) and (IV) we see that $(x, t)$ is not adjacent to any vertex in $D \cap G^{t}$. Thus, in subcase (i) $(x, t)$ has a unique neighbor in $D$. Assume that $t=r$. By (I) there is a unique $y \in V_{r} \cap N(x)$. By definition $(y, r) \in D$ and thus $(x, r)$ is dominated by $D$. Properties (I) and (IV) together imply that $(x, t)$ has no other neighbor in $D$. Finally, assume subcase (iii) holds. By (III) there exists a unique $z \in V_{t} \cap N(x)$. Now $(z, t) \in D$ and $(x, t)$ is dominated by $(z, t)$. Consequently, by (IV) it follows that $(z, t)$ is the only vertex in $D$ that dominates $(x, t)$. We have shown that $D$ is an EOD-set of $G \square K_{m, n}$.

Conversely, suppose that $S$ is an EOD-set of $G \square K_{m, n}$. Since $K_{m, n}$ has diameter 2, we apply Lemma 4.1 and conclude that $\left|S \cap{ }^{g} K_{m, n}\right| \leq 2$ for every vertex $g$ in $G$. We produce a weak partition $\mathcal{C}$ of $V(G)$ as follows. The sets in $\mathcal{C}$ are those in the following specifications. Note that some of these subsets might be empty.

- $V_{0}=\left\{x \in V(G): S \cap{ }^{x} K_{m, n}=\emptyset\right\}$,

- $V_{i}=\left\{x \in V(G): S \cap{ }^{x} K_{m, n}=\{(x, i)\}\right\}$ for $1 \leq i \leq m+n$,

- $V_{[i, m+j]}=\left\{x \in V(G): S \cap{ }^{x} K_{m, n}=\{(x, i),(x, m+j)\}\right\}$ for $1 \leq i \leq m$ and $1 \leq j \leq n$.

The verification that $\mathcal{C}$ is $K_{m, n}$-amenable (that is, it satisfies properties (I)-(V)) follows directly from the assumption that $S$ is an EOD-set of $G \square K_{m, n}$ and is left to the reader.

The graph $G$ in Figure 1 was constructed to have a weak partition that is $K_{2,3}$-amenable. The partite sets of $K_{2,3}$ are as in the development above, $A=\{1,2\}$ and $B=\{3,4,5\}$. For simplicity the vertices of $G$ are labeled to indicate the subset of the weak partition that contains them. For example, the vertices labeled 1 are in $V_{1}$ while the vertex labeled $[2,5]$ is the only member of $V_{[2,5]}$. By Theorem 4.3 the Cartesian product $G \square K_{2,3}$ is an EOD-graph. 


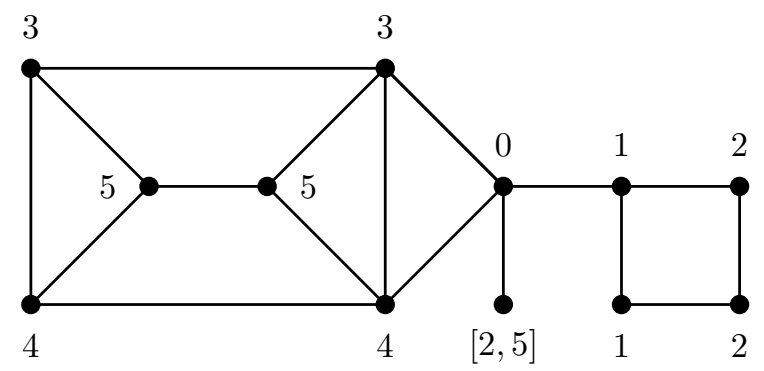

Fig. 1: A $K_{2,3}$-amenable graph $G$

\section{$4.2 G \square C_{r}, r \in\{4,5\}$}

In this subsection we first define a type of weak partition of $V(G)$ that will enable us to characterize those Cartesian products $G \square C_{5}$ that are parallel EOD-graphs with respect to $G$. To describe these weak partitions we need to modify Condition (C) as it was stated in Section 3 and add an additional condition. The operations on the subscripts in these new conditions are made modulo 5 .

$\left(\mathrm{C}^{\prime}\right)\left\langle V_{i} \cup V_{i+1}\right\rangle$ is a matching in $G$ for every $i \in[5]$,

(D) if $x \in V_{i}$, then $\left|N(x) \cap V_{i+2}\right|=1$ and $\left|N(x) \cap V_{i-2}\right|=1$ for every $i \in[5]$.

Notice that the condition $\left(\mathrm{C}^{\prime}\right)$ is weaker than $(\mathrm{C})$. We say that $G$ is $C_{5}$-parallel amenable if there exists a weak partition $\left\{V_{0}, V_{1}, V_{2}, V_{3}, V_{4}, V_{5}\right\}$ of $V(G)$ that satisfies conditions (A), (B), (C') and (D).

Theorem 4.4 For any graph $G$, the Cartesian product $G \square C_{5}$ is a parallel EOD-graph with respect to $G$ if and only if $G$ is a $C_{5}$-parallel amenable graph.

Proof: Assume first that $G$ is a $C_{5}$-parallel amenable graph and let $\left\{V_{0}, V_{1}, V_{2}, V_{3}, V_{4}, V_{5}\right\}$ be a weak partition of $V(G)$ that satisfies conditions (A), (B), (C') and (D). We define a subset $D$ of $V\left(G \square C_{5}\right)$ by $D=\left\{(g, i): g \in V_{i}\right.$ for $\left.i \in[5]\right\}$. Notice that $\left|D \cap{ }^{g} C_{5}\right|=1$ for every $g \in V(G)-V_{0}$. We will show that every vertex of $G \square C_{5}$ is dominated by exactly one vertex of $D$. Let $(g, j)$ be an arbitrary vertex of $G \square C_{5}$.

Assume first that $g \in V_{i}$ for some $i \in[5]$. If $j \in\{i-1, i+1\}$, then $(g, j)$ is dominated by $(g, i)$. Moreover, $(g, j)$ is dominated only by $(g, i)$ in $D$, since $(g, i)$ is the only vertex in $D \cap{ }^{g} C_{5}$ and (B) and $\left(\mathrm{C}^{\prime}\right)$ hold. If $j=i$, then $(g, j)$ is dominated by $\left(g^{\prime}, i\right)$, where $g g^{\prime}$ is an edge in $\left\langle V_{i}\right\rangle$ (notice that $g^{\prime}$ exists by (B)). Note that $(g, j)$ is dominated only by $\left(g^{\prime}, i\right)$ from $D$ by (B) and the fact that $\left|D \cap{ }^{g} C_{5}\right|=1$. It remains to consider $j=i+2$ and $j=i-2$. Assume $j=i+2$; the case $j=i-2$ is similar. By condition (D), $g$ has a unique neighbor $x_{i+2} \in V_{i+2}$. By definition $\left(x_{i+2}, i+2\right) \in D$, and thus $\left(x_{i+2}, i+2\right)$ dominates $(g, j)$. As before, since $\left|D \cap{ }^{g} C_{5}\right|=1$ and since condition (D) holds, it follows that $\left(x_{i+2}, i+2\right)$ is the only vertex of $D$ that dominates $(g, j)$. Hence, if $g \in V_{i}$ for some $i \in[5]$, then $(g, j)$ is dominated exactly once by $D$. Finally, assume that $g \in V_{0}$. By condition (A), $g$ has a unique neighbor $x_{j} \in V_{j}$. This implies that $\left(x_{j}, j\right) \in D$ and that $\left(x_{j}, j\right)$ is the only vertex in $D$ that dominates $(g, j)$. Consequently, $D$ is a parallel EOD-set of $G \square C_{5}$. 
Conversely, let $G \square C_{5}$ be a parallel EOD-graph and let $D$ be a parallel EOD-set with respect to $G$. Let $V_{0}, V_{1}, V_{2}, V_{3}, V_{4}, V_{5}$ be subsets of $V(G)$ defined as follows. If $\left|D \cap{ }^{g} C_{5}\right|=0$, then $g \in V_{0}$. For $i \in[5]$, $g \in V_{i}$ if and only if $\{(g, i)\}=D \cap{ }^{g} C_{5}$. By Lemma 4.1, $\left|D \cap{ }^{g} C_{5}\right| \leq 1$ for every $g \in V(G)$, and thus $\left\{V_{0}, V_{1}, V_{2}, V_{3}, V_{4}, V_{5}\right\}$ is a weak partition of $V(G)$. Note that only $V_{0}$ can be empty.

We will show that this weak partition satisfies conditions (A), (B), (C') and (D). If (A) does not hold, then there exists a vertex $(g, i)$ where $\left|D \cap{ }^{g} C_{5}\right|=0$ and either $\left|N(g) \cap V_{i}\right|=0$ or $\left|N(g) \cap V_{i}\right|>1$ for some $i \in[5]$. In the first case $(g, i)$ is not dominated by $D$ and in the second case $(g, i)$ is dominated by at least two vertices, both contradicting the fact that $D$ is a parallel EOD-set. Thus, (A) holds. If (B) is not satisfied, then there exists $g \in V_{i}$, for some $i \in[5]$, such that either $\operatorname{deg}_{\left\langle V_{i}\right\rangle}(g)=0 \operatorname{ordeg}_{\left\langle V_{i}\right\rangle}(g)>1$, which yields exactly the same contradiction as for (A). Hence, (B) is true as well. If $\left(\mathrm{C}^{\prime}\right)$ does not hold, then there exist $g \in V_{i}$ and $g^{\prime} \in V_{i+1}$ for some $i \in[5]$, such that $g g^{\prime} \in E(G)$. We infer that $(g, i+1)$ is dominated twice, that is by $(g, i)$ and by $\left(g^{\prime}, i+1\right)$, which is not possible. Finally, if (D) does not hold, then for some $i \in[5]$, there exists $x \in V_{i}$ such that $\left|N(x) \cap V_{i+2}\right| \neq 1$ or $\left|N(x) \cap V_{i-2}\right| \neq 1$. Again we get that some vertex is not dominated by $D$ (if $\left|N(x) \cap V_{i+2}\right|=0=\left|N(x) \cap V_{i-2}\right|$ ) or that some vertex is dominated more than once by $D$ (if $\left|N(x) \cap V_{i+2}\right|>1$ or $\left|N(x) \cap V_{i-2}\right|>1$ ), which is not possible. This shows that (D) is also true, which completes the proof.

While the complete characterization of EOD Cartesian products where one factor is $C_{4} \cong K_{2,2}$ was given in Subsection 4.1, here we describe all $G$ such that $G \square C_{4}$ is a parallel EOD-graph with respect to $G$. For $C_{4}$ notice that computations on the subscripts are done modulo 4 in the set [4], and in this case $i+2=i-2$. Thus, we can restate condition (D) as

(D') if $x \in V_{i}$, then $\left|N(x) \cap V_{i+2}\right|=1$ for every $i \in[4]$.

We say that $G$ is $C_{4}$-parallel amenable if there exists a weak partition $\left\{V_{0}, V_{1}, V_{2}, V_{3}, V_{4}\right\}$ of $V(G)$ that fulfills conditions (A), (B), $\left(\mathrm{C}^{\prime}\right)$ and $\left(\mathrm{D}^{\prime}\right)$. The proof of the following theorem follows the same lines as the proof of Theorem 4.4 if we take into consideration computation modulo 4 instead of modulo 5 .

Theorem 4.5 For any graph $G$, the Cartesian product $G \square C_{4}$ is a parallel EOD-graph with respect to $G$ if and only if $G$ is a $C_{4}$-parallel amenable graph.

For $r \in\{4,5\}$ there exist many graphs $G$ which are not $C_{r}$-parallel amenable, but for which $G \square C_{4}$ is an EOD-graph (clearly $G \square C_{4}$ is not a parallel EOD-graph with respect to $G$ in this case). One of the smallest examples is $P_{3}$, which is not $C_{4}$-parallel amenable, but $P_{3} \square C_{4}$ is an EOD-graph, even a parallel EOD-graph with respect to $C_{4}$.

\section{Conclusion}

As already mentioned, this method of defining weak partitions is most easily implemented when one of the graphs has small diameter. Despite this fact, there is no reason why one should not use it on graphs with larger diameter. We illustrate this idea on a special case from the class of cycles.

Our goal is to define a weak partition of a graph $G$ that consists of $V_{0}$ and a family of sets $V_{A}$ where $A$ is a subset of $[k]$ with certain properties. We derive these properties from the second graph in the product, which is $C_{k}$ now. Again we have two possibilities for an edge from $\langle D\rangle$, where $D$ is an EOD-set of $G \square C_{k}$ : either it projects to $C_{k}$ as an edge or as a vertex. If it projects to an edge in $C_{k}$, then $A$ must contain two consecutive elements $i$ and $i+1$. If an edge projects to a single vertex $j \in V\left(C_{k}\right)$, then $j \in A$ 
but neither $j+1$ nor $j-1$ is in $A$. Moreover, two non-consecutive elements of $A$ must differ by at least 3 modulo $k$, so that no vertex in the product is dominated more than once.

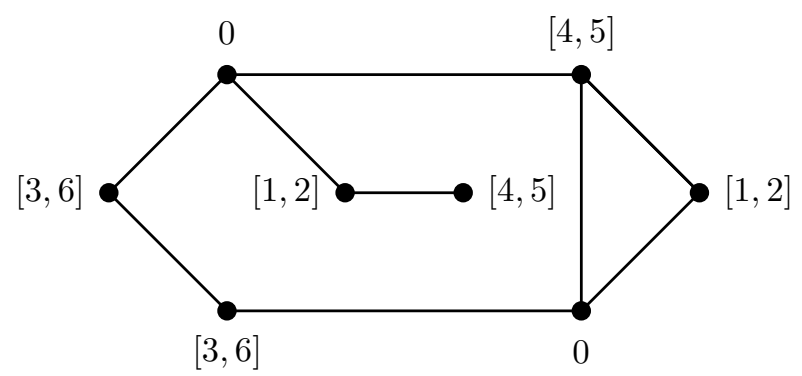

Fig. 2: A " $C_{6}$-amenable" graph $G$

In particular, for $C_{6}$ we obtain the following weak partition:

$$
V_{0}, V_{1}, V_{2}, V_{3}, V_{4}, V_{5}, V_{6}, V_{[1,2]}, V_{[2,3]}, V_{[3,4]}, V_{[4,5]}, V_{[5,6]}, V_{[6,1]}, V_{[1,4]}, V_{[2,5]}, V_{[3,6]} \cdot
$$

Clearly the size of the weak partition increases with $k$. Together with this weak partition, several conditions are needed as well. For instance we need a condition similar to (A) and (V) to care about all vertices from $V_{0}$. As in the case of $K_{2,3}$-amenable graphs, it seems to be hard to decide whether a graph $G$ is a " $C_{k}$-amenable" graph. However, it is not difficult to construct (small) examples of such graphs. An example of a " $C_{6}$-amenable" graph is given in Figure 2. The labeling follows the prescription given for Figure 1.

\section{References}

G. Abay-Asmerom, R. H. Hammack, and D. T. Taylor. Total perfect codes in tensor products of graphs. Ars Combinatoria, 88:129-134, 2008. URL https://www.scopus. $\mathrm{com} /$ inward/record.uri?eid=2-s2 .0 $-47949133881 \&$ partner ID $=40$ \& md5 = $82 \mathrm{cb} 6 \mathrm{dcced} 332 \mathrm{~d} 41 \mathrm{ac} 19 \mathrm{~d} 49 \mathrm{db} 5 \mathrm{afcd} 5 \mathrm{f}$. cited By 4 .

N. Biggs. Perfect codes in graphs. Journal of Combinatorial Theory, Series B, 15(3): 289-296, 1973. doi: 10.1016/0095-8956(73)90042-7. URL https://www.scopus. $\mathrm{com} /$ inward/record. uri ?eid=2-s2.0-0001058806 \&partner $\mathrm{D}=40$ \& md5 = 8578 eda2f5f5ed2464c88eac313b1b54. cited By 84 .

B. Brešar, P. Dorbec, W. Goddard, B. L. Hartnell, M. A. Henning, S. Klavžar, and A. D. F. Rall. Vizing's conjecture: A survey and recent results. Journal of Graph Theory, 69(1):46-76, 2012. doi: 10.1002/jgt.20565. URL https://www.scopus.com/inward/record.uri?eid=2-s2. $0-81955168121$ \&partnerID $=40 \& \mathrm{md} 5=714 \mathrm{c} 9283 \mathrm{eb} 2 \mathrm{c} 834 \mathrm{~b} 7444 \mathrm{bff} 2 \mathrm{f} 12 \mathrm{fafd} 9$. cited By 19.

E. J. Cockayne, B. L. Hartnell, S. T. Hedetniemi, and R. Laskar. Perfect domination in graphs. Journal of Combinatorics, Information and System Sciences, 18:136-148, 1993. 
R. Cowen, S. H. Hechler, J. W. Kennedy, and A. Steinberg. Odd neighborhood transversals on grid graphs. Discrete Mathematics, 307(17-18):2200-2208, 2007. doi: 10.1016/j. disc.2006.11.006. URL https://www.scopus.com/inward/record.uri?eid=2-s2. $0-34447096089$ \&partner ID $=40$ \&md5 $=4$ ef $158 \mathrm{~d} 7008 \mathrm{e} 574 \mathrm{c} 74 \mathrm{c} 14480$ be $62 \mathrm{a} 3 \mathrm{e} 4$. cited By 1.

I. J. Dejter. Perfect domination in regular grid graphs. Australasian Journal of Combinatorics, 42:99-114, 2008. URL https://www.scopus.com/inward/record.uri?eid=2-s2. $0-77956497962 \&$ partner $I D=40 \& \mathrm{md} 5=5 \mathrm{ee} 0089 \mathrm{c} 7962 \mathrm{e} 1 \mathrm{~b} 1 \mathrm{c} 450 \mathrm{~d} 7 \mathrm{cc} 2 \mathrm{c} 7273 \mathrm{fb}$. cited By 7.

P. Dorbec, S. Gravier, S. Klavžar, and S. Špacapan. Some results on total domination in direct products of graphs. Discussiones Mathematicae Graph Theory, 26:103-112, 2006.

H. Gavlas and K. Schultz. Efficient open domination. Electronic Notes in Discrete Mathematics, 11:681-691, 2002. doi: 10.1016/S1571-0653(04)00113-1. URL https: //www.scopus.com/inward/record.uri?eid=2-s2.0-34247126166\&partnerID= $40 \& \mathrm{md} 5=\mathrm{d} 7 \mathrm{e} 81725 \mathrm{fdb} 979 \mathrm{fd} 7 \mathrm{~b} 8 \mathrm{~b} 1 \mathrm{f} 6 \mathrm{db} 56 \mathrm{~b} f \mathrm{f} 30$. cited By 5.

H. Gavlas, K. Schultz, and P. Slater. Efficient open domination in graphs. Scientia. Series A. Mathematical Sciences. New Series, 6:77-84, 2003.

S. Gravier. Total domination number of grid graphs. Discrete Applied Mathematics, 121(1-3): 119-128, 2002. doi: 10.1016/S0166-218X(01)00297-9. URL https://www.scopus . $\mathrm{com} /$ inward/record. uri?eid=2-s2 .0 -84867954416\&partner ID $=40$ \& md5 = 352b54fa6d26558f57889941928b0f01. cited By 21 .

R. Hammack, W. Imrich, and S. Klavžar. Handbook of Product Graphs. CRC Press, Boca Raton, 2012.

M. A. Henning and D. F. Rall. On the total domination number of cartesian products of graphs. Graphs and Combinatorics, 21(1):63-69, 2005. doi: 10.1007/s00373-004-0586-8. URL https: //www.scopus.com/inward/record.uri?eid=2-s2.0-17144421208\&partnerID= 40 \&md5=e3cf8a 8 fce 6 eb 1 fle949164a1db085d4. cited By 13.

P. T. Ho. A note on the total domination number. Utilitas Mathematica, 77:97-100, 2008. URL https: //www.scopus.com/inward/record.uri?eid=2-s2.0-57049085820\&partnerID= $40 \& \mathrm{md} 5=070 \mathrm{fdc} 1064 \mathrm{~b} 24 \mathrm{~b} 409 \mathrm{f} 25 \mathrm{~d} 4185 \mathrm{e} 902 \mathrm{dc} 4$. cited By 6 .

J. Jerebic, S. Klavžar, and S. Špacapan. Characterizing r-perfect codes in direct products of two and three cycles. Information Processing Letters, 94(1):1-6, 2005. doi: 10.1016/ j.ipl.2004.12.010. URL https://www.scopus.com/inward/record.uri?eid=2-s2. $0-13844320862 \&$ partner $I D=40 \& \mathrm{md} 5=89 \mathrm{fb} f 92310 \mathrm{~b} 578653 \mathrm{cba} 376 \mathrm{~d} 19 \mathrm{e} 55152$. cited By 9.

S. Klavžar, S. Špacapan, and J. Žerovnik. An almost complete description of perfect codes in direct products of cycles. Advances in Applied Mathematics, 37(1):2-18, 2006. doi: 10.1016/ j.aam.2005.10.002. URL https://www.scopus.com/inward/record.uri?eid=2-s2. 
$0-33747517241 \&$ partner $I D=40 \&$ md5 $=3 c 94149 \mathrm{a} 146 \mathrm{~d} 61686 \mathrm{~b} 6 \mathrm{clec} 8 \mathrm{eadb} 4966$. cited By 10.

W. F. Klostermeyer and J. L. Goldwasser. Total perfect codes in grid graphs. Bulletin of the Institute of Combinatorics and its Applications, 46:61-68, 2006.

J. Kratochvíl. Perfect codes over graphs. Journal of Combinatorial Theory, Series B, 40(2): 224-228, 1986. doi: 10.1016/0095-8956(86)90079-1. URL https://www.scopus. $\mathrm{com} /$ inward/record.uri ?eid=2-s2.0-38249041657 \&partner ID $=40$ \&m 5 = $2277578 \mathrm{e} 6 \mathrm{~b} 20 \mathrm{a} 40 \mathrm{~d} 30 \mathrm{f} 0 \mathrm{cc} 7 \mathrm{df} 684 \mathrm{f} 1 \mathrm{e} 2$. cited By 22 .

D. Kuziak, I. Peterin, and I. G. Yero. Erratum to total domination number of cartesian products (math. commun., (2004), 9, (35-44)). Mathematical Communications, 19(1): 195-200, 2014a. URL https://www.scopus.com/inward/record.uri?eid=2-s2. $0-84901670740$ \&partner ID $=40 \& \mathrm{md} 5=\mathrm{e} 2 \mathrm{~d} 066 \mathrm{c} 64507 \mathrm{f} 6647 \mathrm{a} 970 \mathrm{ddbe} 9 \mathrm{f} 7 \mathrm{e} 099$. cited By 0 .

D. Kuziak, I. Peterin, and I. G. Yero. Efficient open domination in graph products. Discrete Mathematics and Theoretical Computer Science, 16(1):105-120, 2014b. URL https: //www.scopus.com/inward/record.uri?eid=2-s2.0-84897524357\&partnerID= 40 \&md5=cea 04540 aeb $67 f 59 c 795 b 0$ accce3e554. cited By 1 .

A. McRae. Generalizing NP-completeness Proofs for Bipartite Graphs and Chordal Graphs. Ph.D. Thesis, Clemson University, 1984.

G. Mekišs. Lower bounds for the domination number and the total domination number of direct product graphs. Discrete Mathematics, 310(23):3310-3317, 2010. doi: 10.1016/j. disc.2010.07.015. URL https://www.scopus.com/inward/record.uri?eid=2-s2 . $0-77957272000 \&$ partner $I D=40 \& \mathrm{md} 5=4 \mathrm{fb} 2 \mathrm{bbb} 3709 \mathrm{c} 58 \mathrm{f} 2 \mathrm{e} 2028605439 \mathrm{~b} 2216$. cited By 6 .

D. F. Rall. Total domination in categorical products of graphs. Discussiones Mathematicae Graph Theory, 25:35-44, 2005.

T. Tamizh Chelvam and S. Mutharasu. Efficient open domination in cayley graphs. Applied Mathematics Letters, 25(10):1560-1564, 2012. doi: 10.1016/j.aml.2011.12.036. URL https: //www.scopus.com/inward/record.uri?eid=2-s2.0-84862994859\&partnerID= $40 \& \mathrm{md} 5=588296 \mathrm{~b} 0 \mathrm{e} 0 \mathrm{e} 5 \mathrm{cf} 53 \mathrm{c} 720746 \mathrm{ea} 1 \mathrm{bcdb} 98$. cited By 5 . 